\title{
Immunohistochemical Detection of Skin-Homing T Cells Expressing Fucosyltransferase VII (Fuc-TVII) In Vitro and In Situ
}

\author{
Yoshiko Mizukawa, Kenya Shitara, Yoshimi Yamazaki, Teruhiko Kudo, \\ Hisashi Narimatsu, and Tetsuo Shiohara
}

Departments of Dermatology (YM, YY, TS) and Second Anatomy (TK), Kyorin University School of Medicine, Mitaka, Tokyo; Tokyo Research Laboratories (KS), Kyowa Hakko Kogyo Company, Ltd., Machida, Tokyo; and Division of Cell Biology $(H N)$, Institute of Life Science, Soka University, Hachioji, Tokyo, Japan

\begin{abstract}
SUMMARY: Although expression of cutaneous lymphocyte-associated antigen (CLA) is thought to be a specific marker of skin "homing" T cells, it has become clear that CLA is a good marker for high levels of fucosyltransferase VII (Fuc-TVII) activity but does not necessarily represent the epitope required for binding to E-selectin (Wagers et al, 1996, 1997). Therefore, expression of Fuc-TVII is an attractive candidate for identifying skin "homing" T cells. However, analyses of Fuc-TVII expression in human T cells have been performed only at the mRNA level (Nakayama et al, 2000) because of the lack of mAb. In this study Fuc-TVII was for the first time visualized in individual cells by using a novel mAb that we developed. Double immunofluorescence and immunohistochemistry demonstrated the coexpression of Fuc-TVII and CLA in cell lines in which Fuc-TVII mRNA was shown to be expressed at high levels: whereas CLA expression was seen in the cell membrane, Fuc-TVII was identified in a supra- or perinuclear location. Cytoplasmic Fuc-TVII expression was also detectable in both $\mathrm{CD}^{+}$and $\mathrm{CD} 8^{+} \mathrm{T}_{\text {cells }}$ purified from peripheral blood. Fuc-TVII was also expressed at high levels in many CLA ${ }^{+} \mathrm{T}$ cells infiltrating the skin. In these peripheral T cells, unlike in cell lines, cytoplasmic expression of Fuc-TVII was not always associated with surface CLA expression. This mAb would serve as a valuable tool for selectively identifying a novel subset of skin "homing" T cells that are not detected by the conventional method because of the lack of CLA expression. (Lab Invest 2001, 81:771-773).
\end{abstract}

$F$ human fucosyltransferase VII (Fuc-TVII), eight hybridomas were identified as those secreted antibodies specifically recognizing a 44-kd Fuc-TVII protein in immunoblotting. Immunohistochemistry was performed on several cell lines and peripheral blood $T$ cells, both of which were centrifuged onto microscope slides and tissue specimens, as described previously (Teraki et al, 1994). The slides fixed with 4\% paraformaldehyde (PFA) were incubated with 5\% normal swine serum (NSS; Dakopatts, Glostrup, Denmark) diluted with $0.5 \%$ sodium azide in PBS for 30 minutes. They were then incubated at $4^{\circ} \mathrm{C}$ for 16 hours with Fuc-TVII-specific mAb in permeabilization buffer (0.5\% saponin and $5 \%$ NSS in PBS), and were treated with $3 \% \mathrm{H}_{2} \mathrm{O}_{2}$ for 10 minutes. For efficient intracellular staining of Fuc-TVII, a $70^{\circ} \mathrm{C}$ temperature treatment for 5 minutes was required before staining. Although we attempted a variety of antigen retrieval methods, a combination of heating and permeabilization by saponin was found to stain Fuc-TVII most prominently. The

Received February 23, 2001.

This work was supported by grants from the Ministry of Education, Sports, Science and Culture of Japan to TS.

Address reprint requests to: Dr. Yoshiko Mizukawa, Department of Dermatology, Kyorin University School of Medicine, 6-20-2 Shinkawa, Mitaka, Tokyo 181-8611, Japan.E-mail: tpshio@kyorin-u.ac.jp activity of the primary antibody was revealed by incubating with biotinylated horse anti-mouse immunoglobulin G (IgG) (Vector Laboratories, Burlingame, California) and streptavidin/biotinylated horseradish peroxidase complex (Dakopatts), followed by the visualization of peroxidase activity using 3-amino-9ethylcarbazol (AEC, Dakopatts). Immunoreactivity to Fuc-TVII was detected readily in the cytoplasm of the Fuc-TVII-transfected Namalwa cells, whereas no reactivity was noted in the negative controls, such as Namalwa cells. The staining for Fuc-TVII was found in a supranuclear location, consistent with the Golgi staining pattern. Of the eight mAbs tested, KM1939 gave the most intense staining. To investigate whether Fuc-TVII expression was selectively associated with that of cutaneous lymphocyte-associated antigen (CLA), we initially used double confocal microscopy. The first staining was performed with Fuc-TVII that was visualized by Cy 3-donkey anti-mouse IgG (Jackson ImmunoResearch, West Grove, Pennsylvania), and the second staining with FITC-labeled HECA-452, a mAb for CLA (a kind gift from L. Picker, Department of Pathology, University of Texas Southwestern Medical Center, Dallas, Texas). This experiment confirmed the coexpression of CLA and Fuc-TVII in the same cell, but in a distinct localization (Fig. 1).

We next examined whether Fuc-TVII expression was restricted to a limited $T$ cell subpopulation ex- 
pressing CLA in peripheral blood. For efficient double immunohistochemical staining for Fuc-TVII and CLA in these T cells, the first staining step (Fuc-TVII) with the above protocol was used, and this was followed by the second step (CLA), in which avidin-biotin-alkaline phosphatase complex (ABC-AP) and Vector blue detection system (Vector Laboratories) were used. A substantial proportion of $\mathrm{CLA}^{+}$peripheral blood mononuclear cells (PBMC) were positive for Fuc-TVII in a supranuclear or perinuclear location (Fig. 2A). Representative double staining patterns of purified $\mathrm{CD}^{+}$and $\mathrm{CD}^{+} \mathrm{T}$ cells are shown in Figure 2, B and C. According to the expression patterns of CLA and Fuc-TVII, the $\mathrm{CD}^{+}$and $\mathrm{CD}^{+}{ }^{+} \mathrm{T}$ cells consisted of three populations: $\mathrm{CLA}^{+} \mathrm{Fuc}^{-\mathrm{TVII}^{+}}{ }^{+} \mathrm{CLA}^{+} \mathrm{Fuc}^{-\mathrm{TVII}^{-}}{ }^{-}$, and $\mathrm{CLA}^{-}$Fuc-TVII ${ }^{+}$. These results indicate that expression of CLA and Fuc-TVII is not necessarily induced in a synchronous fashion, although the total percentages of cells expressing each epitope were quite similar. A straightforward interpretation is that both epitopes are expressed in the same $\mathrm{T}$ cells but with different expression kinetics. If so, the Fuc-TVII ${ }^{+}$ $\mathrm{CLA}^{-}$subset would represent early activated cells that eventually differentiate into the Fuc-TVII ${ }^{+} \mathrm{CLA}^{+}$ subset. This possibility was demonstrated in an in vitro culture system (Ryo Takahashi, Yoshiko Mizukawa, Yoshima Yamazaki, and Tetsuo Shiohara, in preparation).

To ascertain whether this mAb was suitable for in situ detection of Fuc-TVII in infiltrating $\mathrm{T}$ cells, skin biopsy specimens obtained from patients with lichen planus, psoriasis vulgaris, and erythema multiforme were assessed by immunohistochemistry. The sections were stained with the above protocol. Double immunohistochemical staining showed that Fuc-TVII was detected in the cytoplasm of CLA-expressing T cells (Fig. 3). Our success would help to open the way for future experiments to identify a novel population of skin "homing" $\mathrm{T}$ cells at the single cell level, both in vitro and in situ, which was impossible by the conventional anti-CLA mAb.

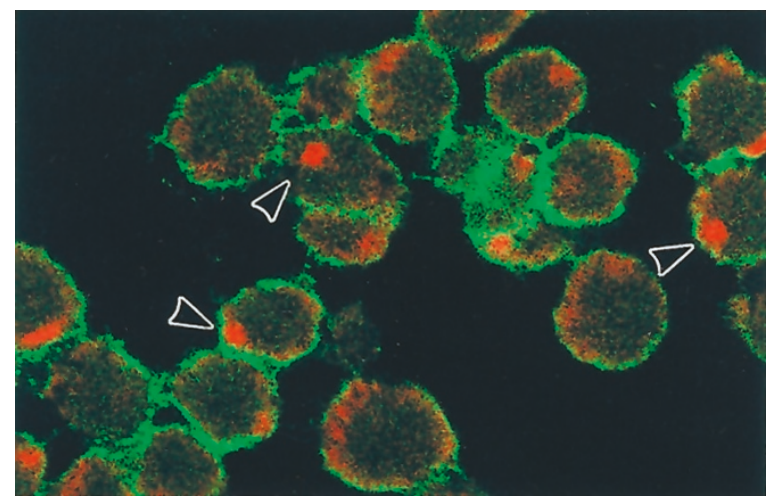

Figure 1.

Confocal microscopic images of the fucosyltransferase VII (Fuc-TVII)transfected Namalwa cells dually immunostained for cutaneous lymphocyteassociated antigen (CLA, green) and Fuc-TVII (red) and visualized using a laser confocal system equipped with an argon/krypton laser (original magnification, $\times 960$ ). CLA expression was seen in the cell membrane, whereas the Fuc-TVII was detected as either diffuse immune reactants or punctate reactants.
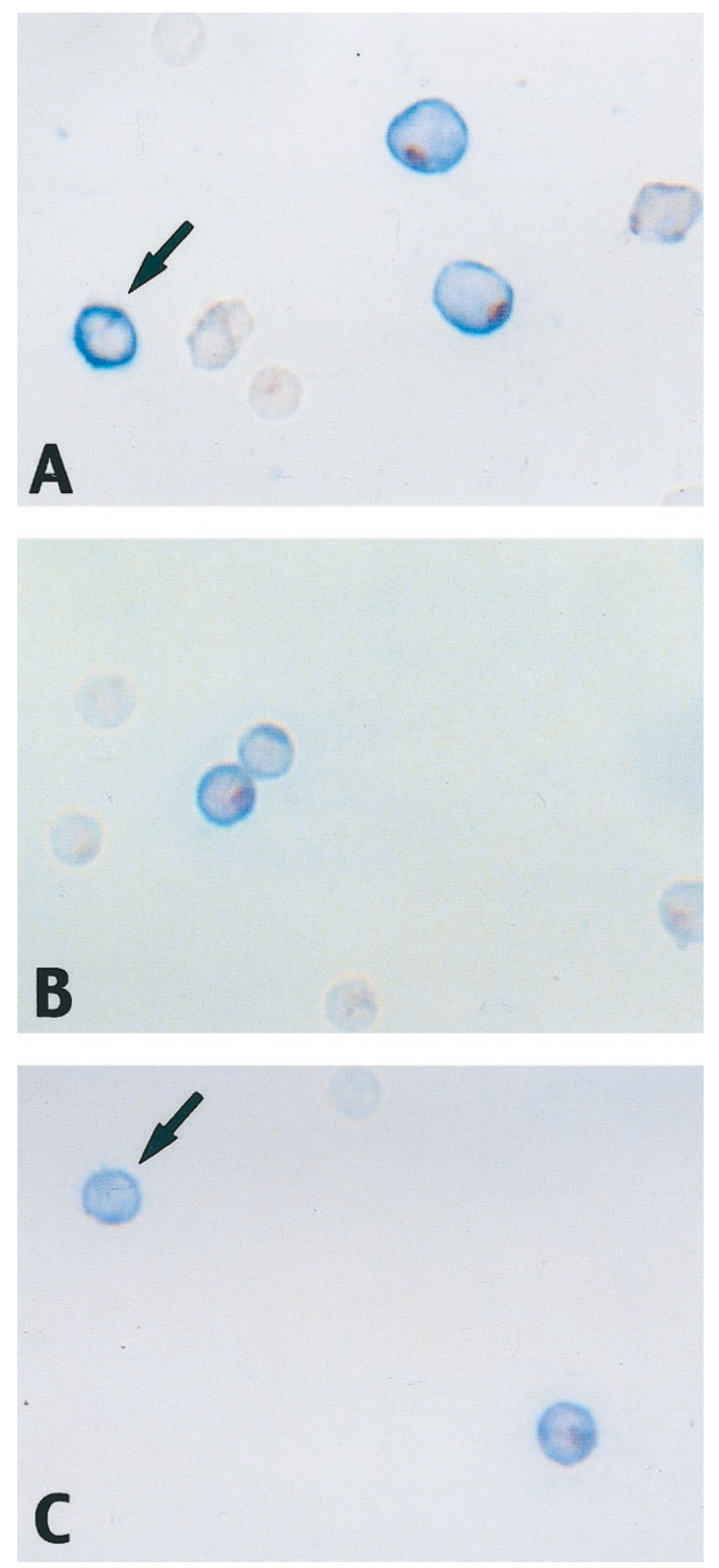

Figure 2.

Double immunohistochemical detection of Fuc-TVII and CLA in peripheral blood mononuclear cells (PBMC) (A) and purified CD4 ${ }^{+}(B)$ and $\mathrm{CD}^{+}(\mathrm{C}) \mathrm{T}$ cells (original magnification, $\times 132$ ). PBMC were obtained from healthy blood donors. Purified $\mathrm{CD}^{+}$and $\mathrm{CD} 8^{+}$T cells were further isolated from the PBMC preparations by negative selection using a human $\mathrm{CD} 4^{+}$or $\mathrm{CD} 8^{+} \mathrm{T}$ cell subset column kit. A, Many CLA ${ }^{+}$(blue) PBMC coexpress cytoplasmic Fuc-TVII (red) in a dot-like pattern. Two CLA ${ }^{+}$Fuc-TVII ${ }^{+}$PBMC appear to be blast-size T cells, because most of $\mathrm{CLA}{ }^{+}$PBMC with a monocyte/macrophage morphologic appearance show relatively intense and diffuse cytoplasmic staining for Fuc-TVII. Intense CLA surface staining is not necessarily associated with cytoplasmic Fuc-TVII staining (arrow). B, Dot-like expression of Fuc-TVII (red) is detected in the cytoplasm of some $\mathrm{CD}^{+}{ }^{+} \mathrm{T}$ cells showing the intense membrane CLA staining (blue). Significant numbers of the CLA-expressing $\mathrm{CD}^{+}{ }^{+} \mathrm{T}$ cells coexpressed Fuc-TVII in a Golgi staining pattern; however, the intensity of cytoplasmic Fuc-TVII staining was not necessarily proportional to that of CLA surface staining. C, Some $\mathrm{CLA}^{+}$(blue) $\mathrm{CD}^{+} \mathrm{T}$ cells coexpress cytoplasmic Fuc-TVII (red) in a dot-like pattern. Discordant expression of CLA and Fuc-TVII is seen in the $\mathrm{CD}^{+}{ }^{+} \mathrm{T}$ cell population (arrow). Compared with $\mathrm{CD}^{+} \mathrm{T}$ cells, fewer $\mathrm{CLA}^{+} \mathrm{CD}^{+} \mathrm{T}$ cells coexpressed Fuc-TVII. 


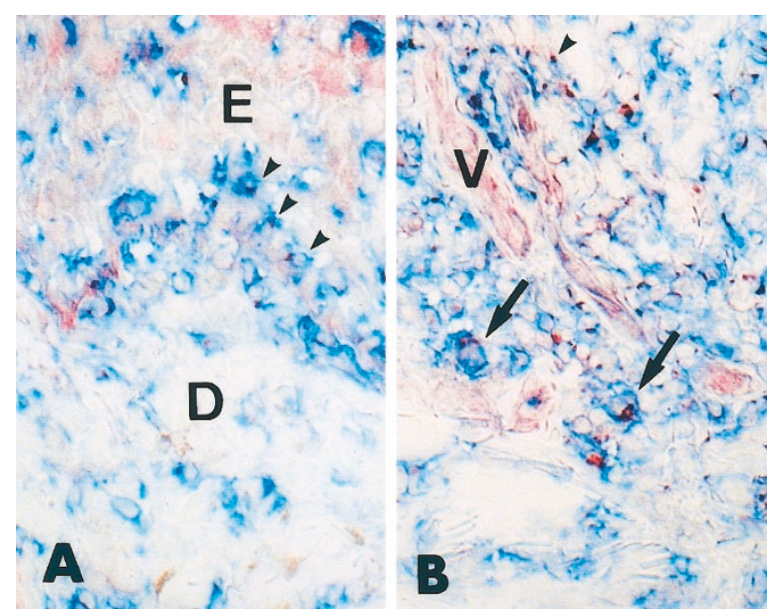

Figure 3.

Double immunohistochemical detection of Fuc-TVII and CLA on infiltrating T cells in biopsy specimens obtained from lichen planus lesions (original magnification, $\times 132)$. A, Scattered T cells, seen at the dermal (D)-epidermal (E) junction, coexpress surface CLA (blue) and cytoplasmic Fuc-TVII (red) (arrowheads). Some CLA ${ }^{+}$cells that do not express Fuc-TVII are also seen. B, Most of infiltrating $T$ cells around blood vessels $(V)$ in the dermis coexpress CLA (blue) and cytoplasmic Fuc-TVII (red), in either a punctate (arrowhead) or a dotlike (arrows) pattern. The percentages of $\mathrm{CLA}^{+} \mathrm{T}$ cells that also coexpressed Fuc-TVII were variable, ranging from $30 \%$ to $50 \%$. CLA- Fuc$\mathrm{TVII}^{+}$cells were hardly detectable in the skin-infiltrating $\mathrm{T}$ cells in situ.

\section{References}

Nakayama F, Teraki Y, Kudo T, Togayachi A, Iwasaki H, Tamatani T, Nishihara S, Mizukawa $\mathrm{Y}$, Shiohara T, and Narimatsu H (2000). Expression of cutaneous lymphocyteassociated antigen regulated by a set of glycosyltransferase in human T cells: Involvement of $\alpha 1,3$-fucosyltransferase VII and $\beta 1,4$-galactosyltransferase I. J Invest Dermatol 115:299306.

Teraki Y, Moriya N, and Shiohara T (1994). Drug-induced expression of intercellular adhesion molecule- 1 on lesional keratinocytes in fixed drug eruption. Am J Pathol 145:550560.

Wagers AJ, Lowe JB, and Kansas GS (1996). An important role for the $\alpha 1,3-$ fucosyltransferase, FucT-VII, in leukocyte adhesion to E-selectin. Blood 88:2125-2132.

Wagers AJ, Stoolman LM, Kannagi R, Craig R, and Kansas GS (1997). Expression of leukocyte fucosyltransferase regulates binding to E-selectin: Relationship to previously implicated carbohydrate epitopes. J Immunol 159:1917-1929. 